In der Rubrik „Literatur kompakt" werden die wichtigsten Originalarbeiten aus der internationalen Fachliteratur referiert.

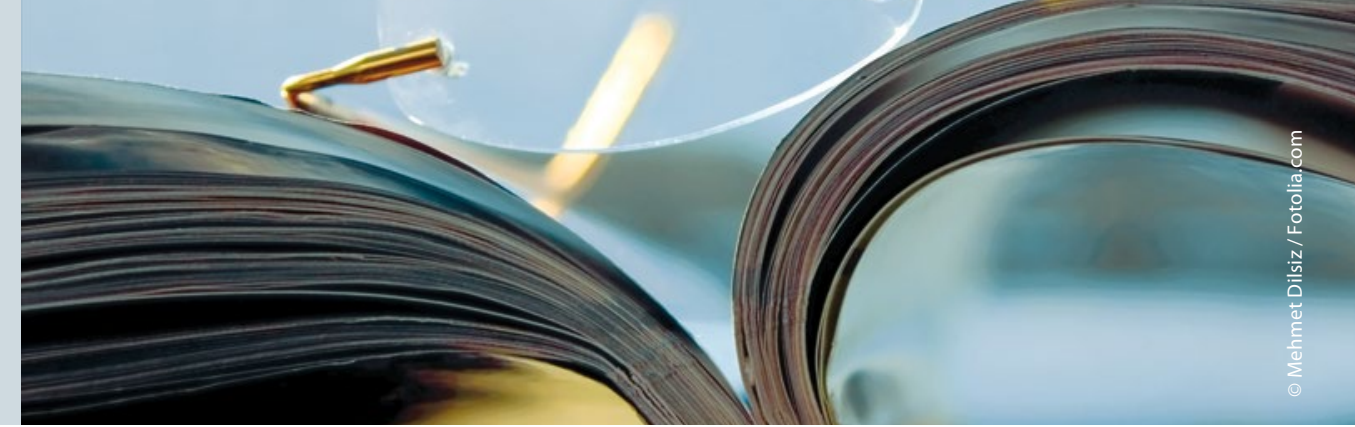

\section{Rheumatoide Arthritis: Regelmäßige Ultraschallkontrollen bringen keine Vorteile}

\author{
Einer schwedischen Studie zufolge ist der systematische Einsatz der Sonogra- \\ fie zur Überwachung von Patienten mit früher rheumatoider Arthritis und \\ leitliniengerechter Therapie nicht gerechtfertigt.
}

$\mathrm{n}$ einigen Studien hat sich gezeigt, dass die klinische Remission einer rheumatioden Arthritis (RA) die Progession des Gelenkschadens nicht unbedingt ausschließt. Mithilfe der Sonografie könnte eventuell auch ein subklinisches Entzündungsgeschehen im Gelenk erkannt werden. Damit ergäbe sich möglicherweise eine neue Kontrollkomponente für die RA-Therapie: die sonografische Progression. Ob die dadurch gewonnenen Erkenntnisse das Ergebnis für den Patienten wirklich verbessern, haben Espen Haavardsholm und sein Team vom Diakonhjemmet Hospital im norwegischen Oslo untersucht.

In die Multicenterstudie ARCTIC (Aiming for Remission in rheumatoid arthritis: a randomised trial examing the benefit of ultrasound in a Clinical Tight Control regimen) wurden zwischen September 2010 und April 2013 insgesamt 238 Patienten zwischen 17 und 75 Jahren eingeschlossen. Alle Probanden erfüllten die Kriterien der „2010 American College of Rheumatology/European League Against Rheumatism classification criteria for rheumatoid arthritis“. Die Patienten erhielten eine adäquate Therapie mit krankheitsmodifizierenden Antirheumatika (DMARD). 116 Studienteilnehmer wurden nach einer konventionelle Strategie engmaschig überwacht, 122 wurden zusätzlich zur klinischen Kontrolle mittels PowerDoppler-Ultraschall untersucht. Im Verlauf von zwei Jahren wurden alle Patienten 13-mal kontrolliert. Die Patienten beider Studienarme wurden nach dem gleichen Schema und mit denselben möglichen Eskalationsstufen medikamentös behandelt. Eine klinische Remission wurde bescheinigt, wenn der Disease Activity Score (DAS 0-10) unter 1,6 Punkten lag und keine Gelenkschwellungen bestanden. Die Therapie im konventionellen Studienarm wurde dem jeweiligen DAS angepasst. Bei der Ultraschallgruppe richtete sich die Eskalation nach dem Sonografiebefund.

Primärer Endpunkt der Studie war eine Kombination aus klinischer Remission und fehlenden Gelenkschwellungen nach 16, 20 und 24 Monaten sowie fehlender radiologischer Progression der Gelenkveränderungen nach 16-24 Monaten. Sekundäre Endpunkte waren Krankheitsaktivität, radiologische Progression, Funktionsfähigkeit, Lebensqualität sowie unerwünschte Ereignisse.

Zwischen den beiden Gruppen zeigten sich im Endergebnis keine nennenswerten Unterschiede: In der Gruppe mit Ultraschallkontrolle erreichten $22 \%$ der Probanden den primären Endpunkt, in der Gruppe mit klinischer Kontrolle $19 \%$. Die sekundären Endpunkte waren in beiden Gruppen ähnlich. Auch bei den schweren unerwünschten Ereignissen unterschieden sich die Probanden wenig (Ultraschallgruppe $5 \%$, konventionelle Gruppe: $6 \%$ ).

Trotz der fehlenden Unterschiede im Ergebnis wurden am Ende der Studie in der Ultraschallgruppe deutlich mehr Patienten mit Biologika behandelt als in der konventionellen Gruppe (29\% versus $17 \%$ ), und weniger Patienten mit Sonografiekontrollen erhielten nach 24 Monaten noch eine Methotrexat-Monotherapie ( $53 \%$ versus $71 \%$ ). In der Ultraschallgruppe hatten 167 Therapieeskalationen und 770 intraartikuläre Kortikoidinjektionen stattgefunden versus 124 Eskalationen und 548 Injektionen im konventionellen Studienarm.

Fazit: Der Nachweis eines subklinischen Entzündungsgeschehens im Ultraschall hat zwar zu vermehrten Therapieeskalationen geführt, diese haben das Gesamtergebnis bei den Patienten aber nicht erkennbar beeinflusst. So kommen Haavardsholm und Kollegen zu dem Schluss, dass eine subklinische Entzündung ohne klinisch erkennbare Krankheitsaktivität letztlich nur von sehr geringer Bedeutung ist. Statt zu nutzen könne die Sonografie sogar eher zu einer Übertherapie führen. Nach Ansicht der Autoren verdeutlicht die ARCTIC-Studie, wie wichtig kontrollierte, randomisierte Studien zur Beurteilung nicht nur von $\mathrm{Me}$ dikamenten, sondern auch von neuen bildgebenden Verfahren sind.

\section{Dr. Christine Starostzik}

Haavardsholm EA et al. Ultrasound in management of rheumatoid arthritis: ARCTIC randomised controlled strategy trial. BMJ 2016; 354: i4205 